\title{
Dynamic Surveillance of Mosquitoes and Their Viromes in Wuhan During 2020
}

\author{
Nanjie Ren 1,2,\#, Shunlong Wang 1,2,\#, Chenyan Shi ${ }^{3}$, Ping $\mathrm{Yu}^{4}$, Lu Zhao ${ }^{1,2}$, Doudou Huang ${ }^{1,2}$, \\ Haixia Ma ${ }^{1,2}$, Shuqi Xiao ${ }^{1,2}$, Fei Wang ${ }^{1,2}$, Zhiming Yuan ${ }^{1,2, *}$ and Han Xia ${ }^{1,2, *}$
}

\begin{abstract}
Objective: Mosquitoes are medically important arthropod vectors that harbor a variety of viruses. Geography and climate are known to be associated with variations in mosquito density, species and viromes. Our study investigated the dynamic changes in mosquito populations, species compositions and viromes in a regularly disinfected environment in Wuhan, China, during 2020.
\end{abstract}

Methods: Traps were set in different mosquito habitats, including an urban residential area, two hospitals, a scenic area and a pig farm in a rural region between April and October of 2020. The collected mosquitoes were subjected to morphological identification, RT-qPCR and metagenomic sequencing.

Results: A total of 2345 adult mosquitoes were collected. Culex mosquitoes were dominant in both urban regions $(90.32 \%, 1538 / 1703)$ and the pig farm $(54.98 \%, 353 / 642)$. In RT-qPCR screening, the prevalence of Banna virus was $15 \%$ and $3 \%$ in mosquitoes from the urban area and the pig farm, respectively, whereas no Japanese encephalitis virus was detected. Culex viromes showed dynamic changes during the collection period. Several mosquito-specific viruses, such as Culex flavivirus, Alphamesonivirus 1, Hubei mosquito virus 2 and Hubei mosquito virus 4, showed seasonal changes and unimodal increases or declines. Other mosquito-specific viruses, such as Wuhan mosquito virus 6, Hubei virga-like virus 2 and Zhejiang mosquito virus 3, were stable in all collected Culex and are potential members of the core viromes.

Conclusion: This study improves understanding of the dynamic composition of mosquito species and the viromes that they carry, and provides useful information for guiding mosquito control and mosquito-borne disease prevention strategies.

Key words: Wuhan, mosquito, virome, surveillance

\section{BACKGROUND}

Mosquitoes are blood-sucking arthropods that have a broad distribution worldwide and can cause severe threats to public health. Mosquitoes are crucial competent vectors for several mosquito-borne viruses (MBVs), such as dengue virus and chikungunya virus, which infect millions of people every year $[1,2]$. The MBVs infecting humans are concentrated in four groups: the Reoviridae (genus Orbivirus and Seadornavirus, such as Tibet orbivirus and Banna virus) the Flaviviridae (genus Flavivirus, such as dengue virus and zika virus), Togaviridae (genus Alphavirus, such as chikungunya virus and Venezuelan equine encephalitis virus) and Bunyavirales (primarily in two families, genus Orthobunyavirus in Peribunyaviridae, such as Bunyamwera
Edited by:

Wanbo Tai, Shenzhen Bay Laboratory

Reviewed by:

Shengyong Feng, Tsinghua University Bo Zhang, Wuhan Institute of Virology, Chinese Academy of Science

The other two reviewers choose to be anonymous

*Corresponding authors: E-mail: yzm@wh.iov.cn (ZY), hanxia@wh.iov.cn (HX)

${ }^{1}$ Key Laboratory of Special Pathogens and Biosafety, Wuhan Institute of Virology, Chinese Academy of Sciences, Wuhan, Hubei, China 2University of Chinese Academy of Sciences, Beijing, China

${ }^{3}$ Center Lab of Longhua Branch and Department of Infectious Disease, The Second Clinical Medical College, Jinan University (Shenzhen People's Hospital), Shenzhen, China

4Shanghai Institute of Hematology, State Key Laboratory of Medical Genomics, Rui Jin Hospital, Shanghai Jiao Tong University School of Medicine, Shanghai, China

\#These authors contributed equally to this work.

Received: July 182021 Revised: September 22021

Accepted: September 72021

Published Online: September 202021 
virus, and some members in the genus Phlebovirus, family Phenuiviridae, such as Rift Valley fever) [3]. In addition to MBVs that can infect both vertebrates and invertebrates, various mosquito-specific viruses (MSVs) have been identified in recent years. These MSVs have potential applications in biological control of mosquito-borne diseases, diagnostic therapies and novel vaccine platforms [4]. MSVs exist in many families, the most common of which are Flaviviridae (genus Flavivirus, such as Culex flavivirus and Quangbinh virus) and Mesoniviridae (entire family, such as Yichang virus and Dianke virus). Notably, the Orthomyxoviridae, whose major representatives are vertebrate viruses, such as influenza viruses [5], are also found in mosquitoes, particularly in the genus Quaranjavirus, such as Quaranfil virus and Johnston Atoll virus [6]. With rapidly developing next-generation sequencing (NGS) technology, viral metagenomics has been used to detect viral diversity and abundance, predict disease outbreaks and identify novel viruses in uncultured mosquito samples [7]. Moreover, viral metagenomic surveillance systems have been established to monitor the viromes derived from mosquito hosts [7].

Hubei is a province located in central China with a suitable environment for mosquito breeding. During the past decade, hundreds of Japanese encephalitis cases have been reported in Hubei [8]. In August 2019, the first local outbreak with a total of 50 cases of dengue fever was reported in Huangzhou, Hubei Province [9]. In addition, two genotypes of Banna virus (BAV) have been isolated from mosquitoes captured in Hubei; this virus is a member of the Seadornavirus genus and may be pathogenic to humans or animals [10]. For MSVs, Quang Binh virus, Culex flavivirus and Yichang virus (YCV), which can decrease the replication of DENV-2 when coinfected in mosquitoes, have also been reported to be present in mosquitoes collected from Hubei $[11,12]$.

Wuhan, the capital city of Hubei Province, has an annual mean temperature of $15.8-17.5^{\circ} \mathrm{C}$ and an annual precipitation of $1150-1450 \mathrm{~mm}$ of rain, thus making this area suitable for mosquito breeding. During the COVID-19 epidemic, in early 2020 , a large-scale disinfection procedure was applied in the environment of the entire city [13]. Therefore, changes in the species or density of mosquito populations may occur in Wuhan with respect to those in previous years. Limited information is available regarding the viral diversity and abundance of mosquitoes in Wuhan. In this study, mosquitoes were collected and classified monthly from representative sites from April to October 2020 , and then dynamic surveillance of the mosquitoes and their viromes was conducted, together with screening for BAV and Japanese encephalitis virus (JEV).

\section{METHODS}

\section{Sampling sites}

Sampling sites were selected in five representative regions of Wuhan. The sampling times were from late April to late October 2020 (SFig 1), as described previously [14].
The sites contain two hospitals, the First People's Hospital of Jiangxia District ( $\left.30^{\circ} 22^{\prime} 23^{\prime \prime} \mathrm{N}, 114^{\circ} 18^{\prime} 56^{\prime \prime} \mathrm{E}\right)$ and

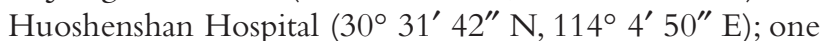
urban residential area, Huanan seafood market $\left(30^{\circ} 37^{\prime} 7^{\prime \prime}\right.$ $\left.\mathrm{N}, 114^{\circ} 15^{\prime} 25^{\prime \prime} \mathrm{E}\right)$; one scenic area, East Lake $\left(30^{\circ} 37^{\prime} 6^{\prime \prime}\right.$ $\mathrm{N}, 114^{\circ} 15^{\prime} 27^{\prime \prime} \mathrm{E}$ ); and one pig farm in a rural region, Huangpi pig farm $\left(30^{\circ} 52^{\prime} 52^{\prime \prime} \mathrm{N}, 114^{\circ} 22^{\prime} 30^{\prime \prime} \mathrm{E}\right)$.

\section{Mosquito collection and sample preparation}

Mosquitoes were collected with light traps with an attractant (Maxttrac, China) set on the shore ponds or in the bushes. Mosquitoes were collected monthly from April to October, except in the pig farm, where trapping occurred only in May because the pig farm was shut down in June. The traps were set from 19:00 to 22:00 pm for 3-7 days every month. Then the mosquitoes were separated, identified and stored at $-80^{\circ} \mathrm{C}$.

The mosquitoes were assigned to different pools according to the collection site, month, species and sex. Each mosquito pool (20 mosquitoes/pool) was triturated with the cryogenic grinding method by using a High-Speed Low-Temperature Tissue Grinding Machine (Servicebio, China) running two 30-second cycles at $50 \mathrm{~Hz}$. After sufficient grinding, $600 \mu \mathrm{L}$ of Roswell Park Memorial Institute (RPMI) medium was added for homogenization [15]. Mosquito macerates were clarified by centrifugation at $10,000 \times \mathrm{g}\left(4^{\circ} \mathrm{C}\right.$ for $\left.30 \mathrm{~min}\right)$ to remove cell debris and bacteria. Supernatants were stored at $-80^{\circ} \mathrm{C}$ until further use.

\section{RNA extraction and RT-qPCR}

RNA was extracted from $200 \mu \mathrm{L}$ of homogenized supernatant sample with Direct-zol RNA MiniPrep (Zymo Research, USA) according to the manufacturer's instructions. The quantitative real-time reverse transcription PCR (RT-qPCR) mixtures for the detection of viral RNA were made with a Luna ${ }^{\circledR}$ Universal Probe One-Step RT-qPCR Kit (New England Biolabs, USA) in accordance with the manufacturer's instructions and then placed in a thermocycler (BIO-RAD CFX96 ${ }^{\text {TM }}$ Real-Time System, USA).

Specific RT-qPCR for BAV and JEV was performed with previously reported primers and probes (STable 1) [10,16,17]. All oligoprimer DNAs were synthesized by TSINGKE (Wuhan Branch, China). Twenty-microliter reaction mixtures containing $5 \mu \mathrm{L}$ of viral RNA and 0.8 $\mu \mathrm{L}$ of each primer were incubated at $55^{\circ} \mathrm{C}$ for $10 \mathrm{~min}$ and $95^{\circ} \mathrm{C}$ for $1 \mathrm{~min}$, followed by 40 cycles of $95^{\circ} \mathrm{C}$ for $10 \mathrm{~s}$ and $55^{\circ} \mathrm{C}$ for $30 \mathrm{~s}$.

\section{Metagenomic sequencing}

The dominant mosquito genera in an urban district and pig farm were selected for sequencing. Briefly, the RNA extracted from each mosquito pool was remixed to form new pools via different strategies. For urban pools, $10 \mu \mathrm{L}$ of RNA extracted from pools from all sampling areas in the same month was mixed into monthly pools. For the Huangpi pig farm pools, $10 \mu \mathrm{L}$ of RNA extracted from pools from the same mosquito genus was mixed into Anopheles or 
Culex pools. The purity and integrity of the RNA pools were verified with a NanoPhotometer ${ }^{\circledR}$ spectrophotometer (IMPLEN, CA, USA), an RNA Nano 6000 Assay Kit and a Bioanalyzer2100 system (Agilent Technologies, CA, USA). One microgram of RNA from each pool was used for library preparation with the NEBNext ${ }^{\circledR}$ Ultra ${ }^{\mathrm{TM}}$ RNA Library Prep Kit for Illumina ${ }^{\circledR}$ (NEB, USA) according to the manufacturer's instructions. Then the libraries were clustered on a cBot Cluster Generation System by using a TruSeq PE Cluster Kit v3-cBot-HS (Illumina) and sequenced with an Illumina NovaSeq 6000 System.

\section{Downstream bioinformatics analysis}

The paired-end reads from NGS were processed with Trimgalore [18] to trim adapters and low-quality bases, and the reads of the host genome (Anopheles or Culex) were discarded with bowtie2 [19] and bedtools [20]. The remaining reads were assembled into contigs with Trinityrnaseq [21]. Contigs longer than $500 \mathrm{bp}$ were filtered and dereplicated (nucleotide identity $>95 \%$ and coverage rate $>80 \%$ ) with CD-HIT [22], and aligned against the nonredundant protein database from NCBI (updated in March 2021) for taxonomic classification by using Diamond BLASTX [23]. The BLASTX results were processed with the LCA algorithm (weighted LCA percentage $=75 \%$, e-value $=1 \times 10^{-5}$, minimum support $=1$ ) with MEGAN [24]. Taxonomic classification was conducted mainly at the family level. The trimmed reads were aligned to the contigs of each sample with checkm [25] to calculate the abundance of eukaryotic viral contigs. Data visualization was conducted with ComplexHeatmap [26] and the ggplot2 [27] package in R [28]. A viral species that contained more than 500 reads was considered to be present in a pool.

\section{RESULTS}

\section{Mosquito composition}

The temperature and precipitation from April to October in Wuhan during 2020 (Fig 1A) were recorded. The rainfall increased above the 10-year average data in June and July, but the average temperature was lower than that in previous years [29].

From April to October, a total of 2345 adult mosquitoes were collected: 1703 from the urban area and 642 from the rural pig farm. The seasonal fluctuations in mosquito density in the urban area revealed a bimodal pattern, with the first peak occurring in May or June, and the second significantly lower peak occurring in September or October (Fig 1B and E), similarly to previous data [30].

Four genera of mosquitoes were classified: Culex, Aedes, Anopheles and Armigeres (Fig 1C and D). In these four genera, the predominant species in both the urban area and pig farm was Culex, in agreement with existing reports [30]. In the urban area, Culex mosquitoes composed a large proportion (90.32\%, 1538/1703), followed by Aedes (9.21\%, 157/1703). Both Anopheles $(0.18 \%, 3 / 1703)$ and Armigeres $(0.18 \%, 3 / 1703)$ were caught in small amounts.
However, in the pig farm, the main mosquito species were Culex (54.98\%, 353/642) and Anopheles (43.77\%, 281/642). Armigeres $(1.25 \%, 8 / 642)$ was much less collected, and no Aedes mosquitoes were caught.

\section{BAV and JEV detection by RT-qPCR}

A total of 1507 of the 1703 mosquitoes from the urban area were separated into 90 pools, and 634 of the 642 mosquitoes from the pig farm were separated into 33 pools. In the mosquitoes from the urban area, 15\% (14/90 pools) were positive for BAV according to RT-qPCR (Ct values ranging from 32 to 38), and the BAV-positive samples were mainly distributed in residential areas from May to July. In the mosquitoes from the pig farm, $3 \%(1 / 33$ pools, $\mathrm{Ct}=32.71)$ were positive for BAV. However, none of the mosquitoes were JEV positive (STable 2).

\section{Virome profiles of mosquitoes collected in Wuhan}

The seven Culex monthly pools (whct-C_04 to whct-C_10) from the urban area and one Anopheles (hpzc-A_05) and one Culex pool (hpzc-C_05) from the Huangpi pig farm were processed for metagenomic sequencing. All nine pools contained a total of 53,983,154 host-genome-removed reads (Table 1). A total of 2,663,238 de novo assembled contigs were added. The contigs were clustered and dereplicated (longer than $500 \mathrm{bp}$, nucleotide identity > 95\% and coverage $>80 \%$ ) into 413,645 nonredundant contigs. With BLASTX, 1189 and 412,456 contigs were aligned to eukaryotic viruses and other organisms, respectively.

The viromes of the collected mosquitoes were classified into 20 viral families and a group of unclassified viruses (Fig $2 \mathrm{~A})$. Invertebrate viruses were dominant in the viromes, and the hosts of 11 viruses remain unknown (Fig 2B). Only Hubei chryso-like virus 1 (HCLV1) has a vertebrate host.

The abundance of each viral species found in at least one pool containing more than 500 reads is shown in the heatmap (Fig 3). The viromes contained 52 viral species in total. Thirty of them were classified into 20 viral families, and the other 22 viruses lacked accurate taxonomic classification. Unclassified viruses composed a large proportion of the viromes in all pools.

In urban districts, 18 viral families were present in the viromes of Culex mosquito pools. The viral diversity in urban districts changed by month and showed two peaks. The highest viral diversity occurred in the pool whct-C_06 (Fig 3). At the family level, Orthomyxoviridae and Flaviviridae were the dominant viral families and were stably present in the monthly pools (Fig 3). The relative abundance of Orthomyxoviridae fluctuated from $5.91 \%$ to $38.37 \%$ during the collection time, whereas Flaviviridae showed high relative abundance $(26.47 \%-47.87 \%)$ from August to October. At the viral species level, viruses with unimodal changes in relative abundance (the maximum difference $\geq 20 \%$ ) were regarded as viruses with dynamic changes. Culex flavivirus (family Flaviviridae) showed low abundance in April to July and higher abundance in September to October. Alphamesonivirus 1 (family Mesoniviridae), Hubei mosquito 
A

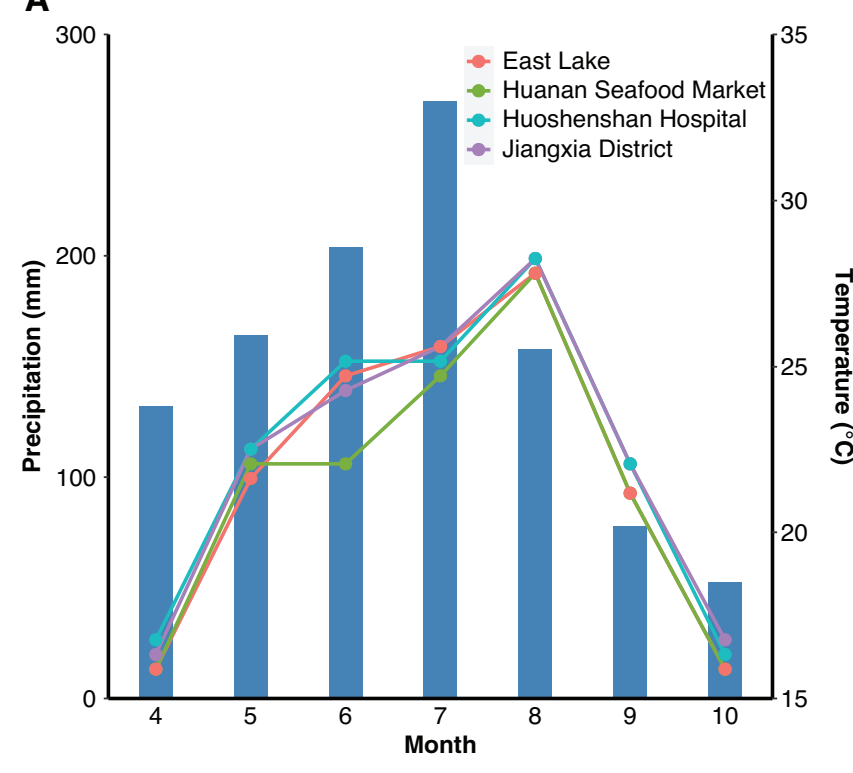

C

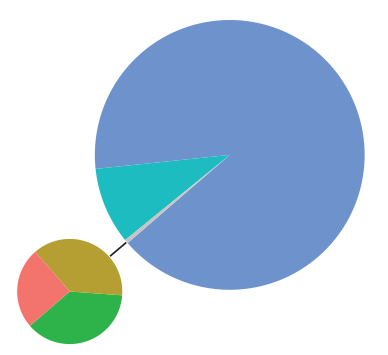

D

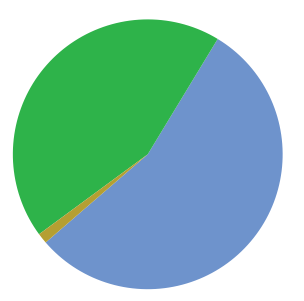

B

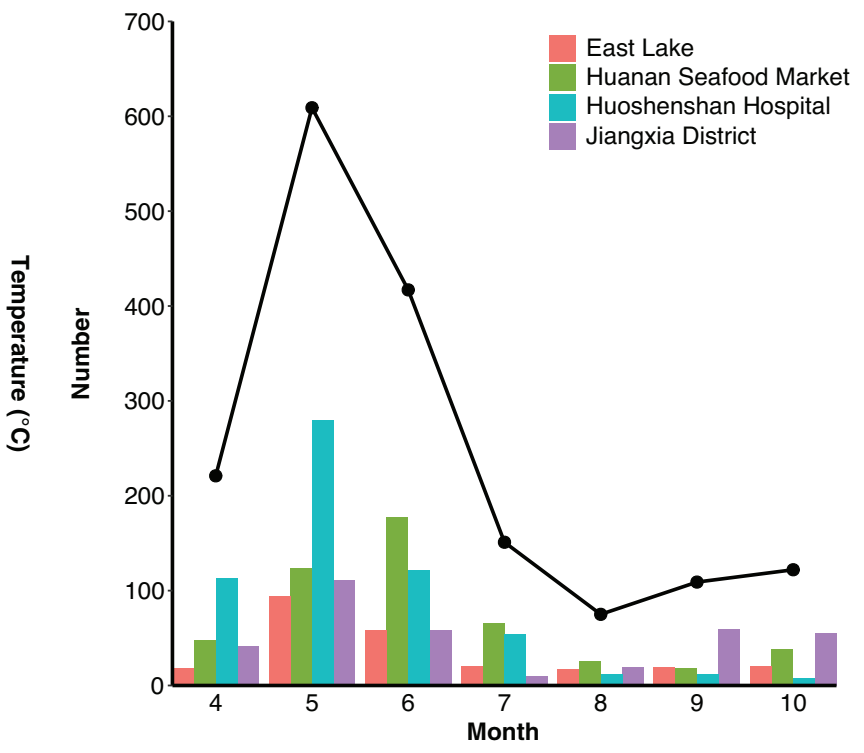

E

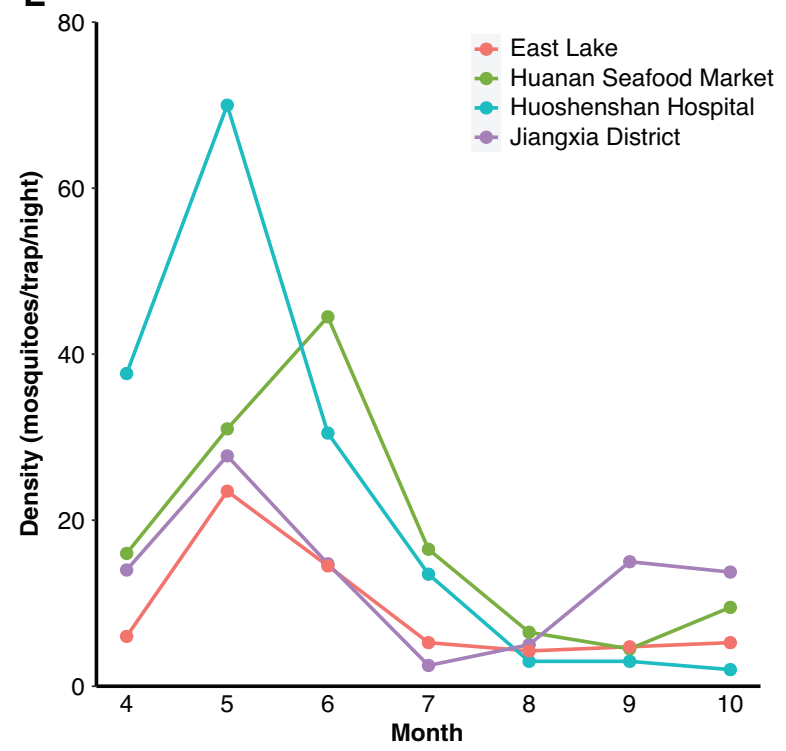

FIGURE 1 | The species and amounts of mosquitoes collected in urban districts and the Huangpi pig farm in Wuhan from April to October of 2020.

(A) The histograms and lines show the precipitation and average temperature of each month, respectively. (B) The mosquitoes collected in urban districts per month. The line shows the total mosquitoes per month. (C) The proportion of mosquito genera in urban districts. (D) The proportion of mosquito genera in the Huangpi pig farm. (E) The abundance of mosquitoes at different collection times.

virus 2 (unclassified family) and Hubei mosquito virus 4 (unclassified family) showed unimodal curves, and their peaks occurred in May, August and June, respectively.

In the Huangpi pig farm, the Culex pool, which contained 13 viral families and 4 unique viruses (Atrato Sobemo-like virus 4, Yongsan sobemo-like virus 1, Primus virus and Ochlerotatus-associated narna-like virus), had viromes similar to those of Culex from urban districts, but differences in viral composition were detected in the families of Parvoviridae and Circoviridae. The diversity of viromes in Anopheles mosquitoes was significantly lower than that in Culex mosquitoes. The Anopheles pool had six unique viruses, and the most abundant virus was Xincheng anphevirus. Six viral species (Wuhan mosquito virus 5, Atrato virus, Chibugado virus, Hubei Beny-like virus 1, Hubei odonate virus 15 and Aedes alboannulatus orthomyxo-like virus) were found in both Culex and Anopheles from the Huangpi pig farm, four of which (Wuhan mosquito virus 5, Atrato virus, Hubei Benylike virus 1 and Aedes alboannulatus orthomyxo-like virus) were absent in Culex mosquitoes in urban districts (Fig 3). 
TABLE 1 | Dominant mosquitoes in Wuhan and metagenomic sequencing.

\begin{tabular}{lllllll}
\hline Sampling site & Sample name & $\begin{array}{l}\text { Mosquito } \\
\text { genus }\end{array}$ & $\begin{array}{l}\text { Month of } \\
\text { collection }\end{array}$ & $\begin{array}{l}\text { Number of Mosquitoes } \\
\text { for sequencing }\end{array}$ & $\begin{array}{l}\text { Host-Genome-removed } \\
\text { reads (viral proportion) }\end{array}$ & $\begin{array}{l}\text { Number of Nonredundant } \\
\text { contigs aligned to eukaryotic } \\
\text { viruses }\end{array}$ \\
\hline Urban district & whct-C_04 & Culex & April & 220 & $4,765,707(5.90 \%)$ & 108 \\
& whct-C_05 & Culex & May & 600 & $5,115,257(19.26 \%)$ & 176 \\
& whct-C_06 & Culex & June & 339 & $8,763,700(11.46 \%)$ & 211 \\
& whct-C_07 & Culex & July & 109 & $4,180,592(12.96 \%)$ & 84 \\
& whct-C_08 & Culex & August & 31 & $11,091,085(3.50 \%)$ & 45 \\
whct-C_09 & Culex & September & 97 & $3,614,845(10.60 \%)$ & 63 \\
whct-C_10 & Culex & October & 111 & $2,988,426(6.02 \%)$ & 84 \\
Huangpi pig farm & hpz-A_05 & Anopheles & May & 281 & $1,677,474(20.14 \%)$ & 137 \\
& hpzc-C_05 & Culex & May & 353 & $11,786,068(3.37 \%)$ & 281 \\
\hline
\end{tabular}

\section{A}

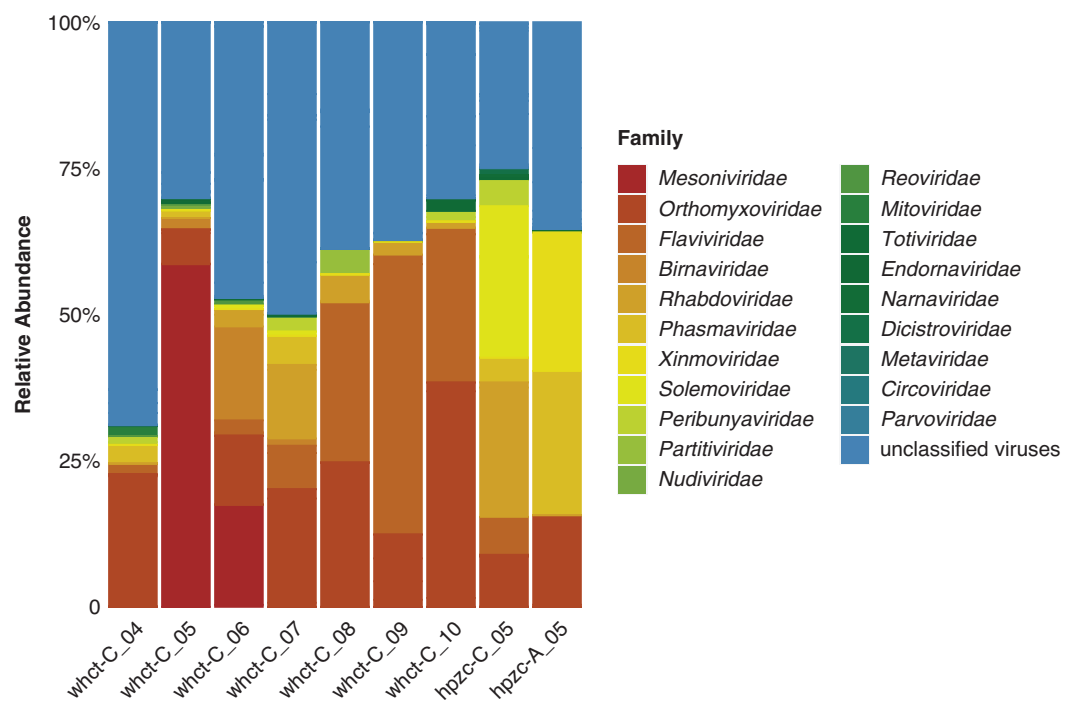

B

FIGURE 2 | Families and hosts of viruses in the viromes of mosquitoes in different pools.

(A) The map shows the relative abundance of viral families in each pool. (B) The composition of the hosts of all 52 viral species.

\section{DISCUSSION}

In this study, we performed 7 months of mosquito monitoring in Wuhan during 2020 to determine the dynamic changes in mosquito populations and their viromes. The main mosquito species and the patten of seasonal fluctuation of mosquito density in 2020 were similar to those in surveys in Wuhan during 2017 to 2019 [9]. However, a decrease in the mosquito population was observed, possibly because of frequent environmental cleaning and disinfection in the city.

During BAV and JEV surveillance, RNA of BAV was detected by RT-qPCR in several mosquito pools, but no contigs associated with BAV were found in the NGS sequencing. This finding may be explained by the following. First, all RNA from pools per month were mixed before NGS, and most pools were BAV-negative, thereby potentially diluting and weakening the signal. Second, the RNA copies of BAV-positive pools were low (Ct values ranging from 32 to 38), and RNA library preparation steps can also lead to loss of low abundance RNAs. Although pigs are the main amplifying host of JEV in nature, JEV was not found in the pig farm. One possible explanation is that we sampled mosquitoes from the pig farm were only in May, and mosquitoes in July and August were not analyzed.

Viral metagenomics has been applied in the analysis of viromes from mosquitoes in many studies [31-33]. Culex species from urban districts and the pig farm had similar viral family compositions. Rhabdoviridae was most prevalent in the pig farm, and several unclassified viruses existed in only one of the two locations, probably because of the diverse mosquito hosts and environments in urban and rural areas [34]. Wuhan mosquito orthophasmavirus 1 (family Phasmaviridae) 


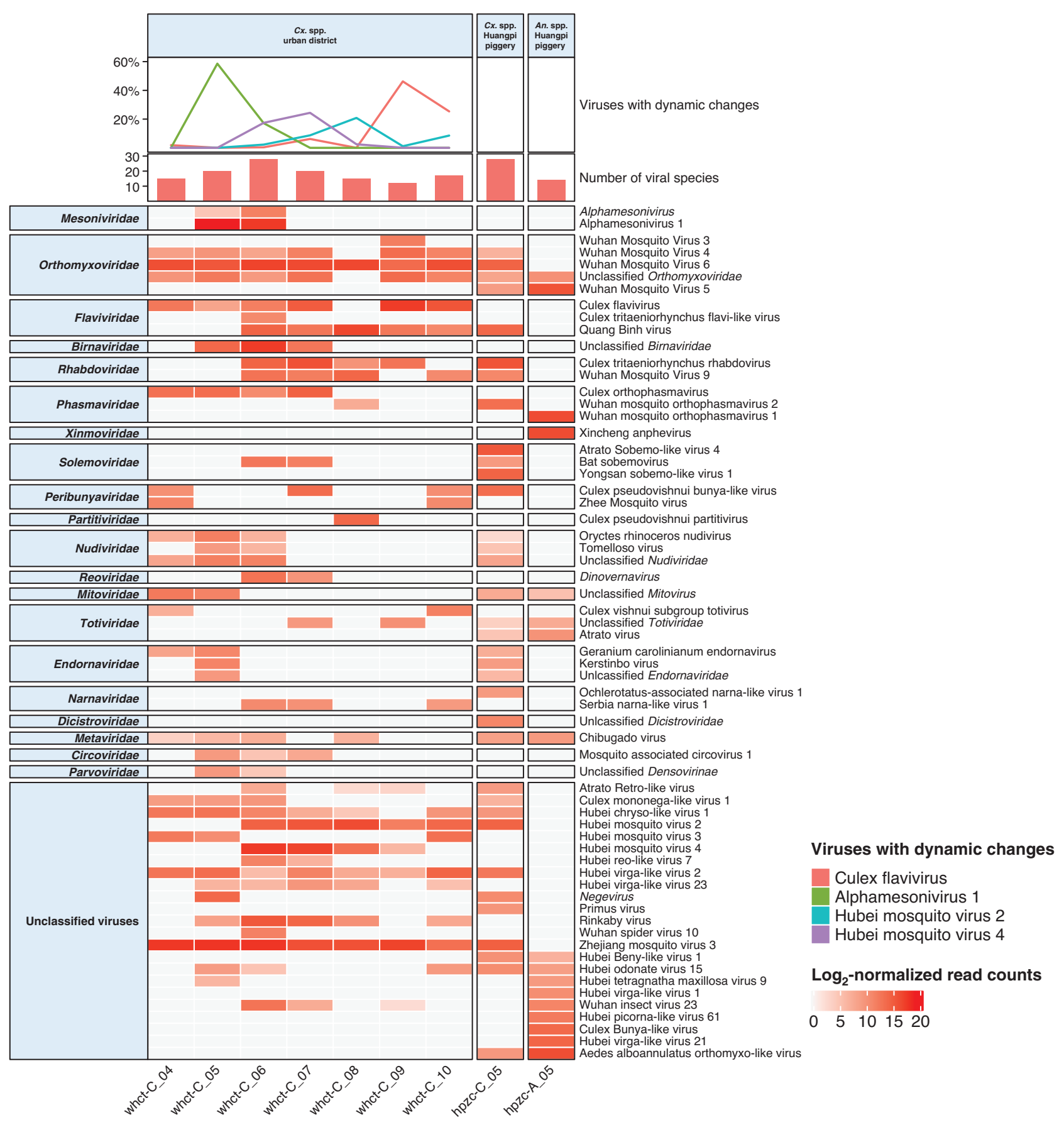

FIGURE 3 | The abundance of viruses in each pool.

The viral species containing more than 500 reads in at least one pool. The bar plot shows the number of viral species in each sample. The line plot at the top shows four viruses that had dynamic changes at different collection times.

and Xincheng anphevirus (family Xinmoviridae) dominated in Anopheles, both of which are MSVs. Previous studies in Guadeloupe and Japan have reported significant differences in the compositions of viromes from different mosquito species, owing to their different biological habits, which may bring them into contact with different environmental viromes $[33,35]$. We also found that Anopheles and Culex had significantly distinct viral diversity.

Several studies have reported that viruses such as Guadeloupe mosquito virus, Phasi charoen-like phasivirus and Guadeloupe Culex rhabdovirus form the "core viromes" of mosquitoes (loosely defined as a set of viruses found in most individuals in a particular mosquito population or a set of viruses found in mosquito species from multiple geographical regions) $[33,36]$. In our study, Wuhan mosquito virus 6, Hubei virga-like virus 2 and Zhejiang mosquito virus 3 were present in Culex from both districts (Fig 3), thus suggesting that they are potential members of the core viromes of Culex in Wuhan. However, further studies are needed to explore their function. 
In a study performed in Yichang (Hubei Province, 289 kilometers from Wuhan) in 2017, the dominant viral families in Culex were Herpesviridae and Adenoviridae with vertebrate hosts [31]. In our study, Wuhan mosquito viruses 3, 4, 5 and 6 (in the genus Quaranjavirus, family Orthomyxoviridae), and Culex flavivirus, Culex tritaeniorhynchus flavi-like virus (CtFLV) and Quang Binh virus (in the Flaviviridae) were prevalent with high abundance in Culex viromes in 2020. The ecological and environmental differences between Yichang and Wuhan might have influenced the mosquito virome compositions. Furthermore, a considerable portion of female mosquitoes collected in Yichang were bloodengorged, thus indicating that the viruses from vertebrate hosts were detected.

Several members of Quaranjavirus, such as Quaranfil virus and Johnston Atoll virus, can infect both vertebrate and invertebrate hosts, and result in the death of newborn mice in the laboratory [6]. However, Wuhan mosquito viruses 3, 4, 5 and 6 have not been properly characterized, and whether they are potentially pathogenic to humans and livestock remains to be confirmed. Quang Binh virus was first isolated in Vietnam and then chronologically reported in Yunnan, Hubei and northwestern China [31,32,37]. Many Culex flavivirus strains have been reported in Culex from different parts of the world. Interestingly, Culex flavivirus and West Nile virus co-infection in mosquitoes are positively correlated $[38,39]$. CtFLV has recently been identified from the local Culex in Japan and is closely associated with Shayang fly virus 4, which was previously identified in Hubei Province. However, neither has been characterized [35], and this is the first report of CtFLV detection in Wuhan districts.

MSVs have shown seasonal activity in mosquito virome studies in the USA and Trinidad [40]. The dynamic changes in the viromes of Culex from urban districts in Wuhan were monitored (Fig 3). The viral diversity in the monthly pools followed a bimodal distribution, with the two peaks occurring in June and October. Culex flavivirus, Alphamesonivirus 1, Hubei mosquito virus 2 and Hubei mosquito virus 4 were highly abundant for several months but disappeared in the other months, thus suggesting that they might be strongly correlated with the changes in seasons and might be associated with the activity of the Culex population.

\section{CONCLUSIONS}

Our results showed the dynamic changes in mosquito composition and viromes under a continual disinfection regime, thus providing fundamental data for local vector and arbovirus prevention and control.

\section{FUNDING}

This work was supported by the Wuhan Science and Technology Plan Project (2018201261638501) and Open Foundation of Key Laboratory of Tropical Translational Medicine of Ministry of Education, Hainan Medical University (2021TTM010).

\section{COMPETING INTERESTS}

The authors declare that there are no conflicts of interest.

\section{DATA AVAILABILITY}

All raw sequencing data are available from the NCBI SRA database with accession numbers SRR15661508, SRR15661507, SRR15661506, SRR15661505, SRR15661504, SRR15661503, SRR15661502, SRR15661501 and SRR15661500. The BioProject accession for the RNA-seq raw data is PRJNA758176.

\section{REFERENCES}

1. Souza-Neto JA, Powell JR, Bonizzoni M. Aedes aegypti vector competence studies: a review. Infect Genet Evol. 2019;67:191-209.

2. Arcà B, Colantoni A, Fiorillo C, Severini F, Benes V, Di Luca M, et al. MicroRNAs from saliva of anopheline mosquitoes mimic human endogenous miRNAs and may contribute to vector-hostpathogen interactions. Sci Rep. 2019;9:2955.

3. Braack L, Gouveia De Almeida AP, Cornel AJ, Swanepoel R, De Jager $C$. Mosquito-borne arboviruses of African origin: review of key viruses and vectors. Parasites and Vectors. 2018;11:29.

4. Atoni E, Zhao L, Karungu S, Obanda V, Agwanda B, Xia H, et al. The discovery and global distribution of novel mosquitoassociated viruses in the last decade (2007-2017). Rev Med Virol. 2019;29:e2079.

5. Nelson PP, Papadopoulos NG, Skevaki C. Respiratory viral pathogens. 2nd ed. Elsevier Inc., 2020.

6. Presti RM, Zhao G, Beatty WL, Mihindukulasuriya KA, da Rosa AP, Popov VL, et al. Quaranfil, Johnston Atoll, and Lake Chad Viruses are novel members of the family orthomyxoviridae. J Virol. 2009;83:11599-11606.

7. Brinkmann A, Nitsche A, Kohl C. Viral metagenomics on bloodfeeding arthropods as a tool for human disease surveillance. Int J Mol Sci. 2016;17:1743.

8. Zou W, Zhan J, Luo Y. Analysis of laboratory confirmed case of epidemic encephalitis B in hubei province from 2011 to 2016. China Heal Ind. 2017;14:14-16.

9. Huang D, Liu L, Chen Q, Wu Y, Liu M, Xiong J. Analysis of dengue epidemic and Aedes vector surveillance in Hubei province, China, 2019. Chin J Vector Biol Control. 2021;32: 38-40 [in Chinese].

10. Xu LH, Cao YX, He LF, Wang HQ, He Y, Fu SH, et al. Detection of Banna virus-specific nucleic acid with TaqMan RT-PCR assay. Chinese J Exp Clin Virol. 2006;20:47-51.

11. Wang $Y$, Xia H, Zhang B, Liu X, Yuan Z. Isolation and characterization of a novel mesonivirus from Culex mosquitoes in China. Virus Res. 2017;240:130-139.

12. Atoni $E$, Zhao L, Hu C, Ren $N$, Wang $X$, Liang $M$, et al. A dataset of distribution and diversity of mosquito-associated viruses and their mosquito vectors in China. Sci Data. 2020;7:342.

13. Zhou P, Shi Z. SARS-CoV-2 spillover events. Science. 2021;371:120-122.

14. Zhao L, Atoni E, Nyaruaba R, Du Y, Zhang H, Donde O, et al. Environmental surveillance of SARS-CoV-2 RNA in wastewater systems and related environments in Wuhan: April to May of 2020. J Environ Sci. 2022;112:115-120.

15. Wang J, Li H, He Y, Zhou Y, Xin A, Liao D, et al. Isolation of Tibet orbivirus from Culicoides and associated infections in livestock in Yunnan, China. Virol J. 2017;14:105.

16. Pyke AT, Smith IL, Van Den Hurk AF, Northill JA, Chuan TF, Westacott AJ, et al. Detection of Australasian Flavivirus encephalitic viruses using rapid fluorogenic TaqMan RT-PCR assays. J Virol Methods. 2004;117:161-167.

17. Pyke AT, Williams DT, Nisbet DJ, van den Hurk AF, Taylor CT, Johansen CA, et al. The appearance of a second genotype of 
Japanese encephalitis virus in the Australasian region. Am J Trop Med Hyg. 2001;65:747-753.

18. Babraham Bioinformatics. Trim Galore! 2012. https://www. bioinformatics.babraham.ac.uk/projects/trim_galore/.

19. Langmead B, Salzberg SL. Fast gapped-read alignment with Bowtie 2. Nat Methods. 2012;9:357-359.

20. Quinlan AR. BEDTools: The Swiss-Army tool for genome feature analysis. Curr Protoc Bioinforma. 2014;2014:11.12.1-11.12.34.

21. Haas BJ, Papanicolaou A, Yassour M, Grabherr M, Blood PD, Bowden J, et al. De novo transcript sequence reconstruction from RNA-seq using the Trinity platform for reference generation and analysis. Nat Protoc. 2013;8:1494-1512.

22. Fu L, Niu B, Zhu Z, Wu S, Li W. CD-HIT: accelerated for clustering the next-generation sequencing data. Bioinformatics. 2012;28:3150-3152

23. Buchfink B, Reuter K, Drost HG. Sensitive protein alignments at tree-of-life scale using DIAMOND. Nat Methods. 2021;18:366-368.

24. Huson DH, Beier S, Flade I, Górska A, El-Hadidi M, Mitra S, et al. MEGAN community edition - interactive exploration and analysis of large-scale microbiome sequencing data. PLoS Comput Biol. 2016;12:e1004957.

25. Parks DH, Imelfort M, Skennerton CT, Hugenholtz P, Tyson GW. CheckM: Assessing the quality of microbial genomes recovered from isolates, single cells, and metagenomes. Genome Res. 2015;25:1043-1055.

26. Gu Z, Eils R, Schlesner M. Complex heatmaps reveal patterns and correlations in multidimensional genomic data. Bioinformatics. 2016;32:2847-2849.

27. Wickham H. ggplot2: elegant graphics for data analysis. New York: Springer-Verlag, 2016.

28. Team C. R: A Language and Environment for Statistical Computing. 2015.

29. National Greenhouse Data System. 2020. http://data. sheshiyuanyi.com/WeatherData/.

30. Liu J, Mao W, Ding H, Li H, Chen L, Chen X, et al. Composition and diversity of mosquito community in Wuhan from 2017 to 2019. J Cent China Norm Univ. 2021;55:416-423.
31. Atoni E, Wang Y, Karungu S, Waruhiu C, Zohaib A, Obanda V, et al. Metagenomic virome analysis of Culex mosquitoes from Kenya and China. Viruses. 2018;10:30.

32. He X, Yin Q, Zhou L, Meng L, Hu W, Li F, et al. Metagenomic sequencing reveals viral abundance and diversity in mosquitoes from the Shaanxi-Gansu-Ningxia region, China. PLoS Negl Trop Dis. 2021;15:e0009381.

33. Shi C, Beller L, Deboutte W, Yinda KC, Delang L, Vega-Rúa $A$, et al. Stable distinct core eukaryotic viromes in different mosquito species from Guadeloupe, using single mosquito viral metagenomics. Microbiome. 2019;7:121.

34. Guo H, Li H, Wu L, Ding H, Liu J, Wu T, et al. The structure, spaciotemporal dynamics, and diversity of mosquito communities in Wuhan. Chinese J Appl Entomol. 2020;57:955-962.

35. Faizah AN, Kobayashi D, Isawa H, Amoa-Bosompem M, Murota K, Higa Y, et al. Deciphering the virome of culex vishnui subgroup mosquitoes, the major vectors of japanese encephalitis, in Japan. Viruses. 2020;12:264.

36. Shi C, Zhao L, Atoni E, Zeng W, Hu X, Matthijnssens J, et al. Stability of the virome in lab- and field-collected Aedes albopictus mosquitoes across different developmental stages and possible core viruses in the publicly available virome data of aedes mosquitoes. MSystems. 2020;5:e00640-20.

37. Crabtree MB, Nga PT, Miller BR. Isolation and characterization of a new mosquito flavivirus, Quang Binh virus, from Vietnam. Arch Virol. 2009;154:857-860.

38. Guggemos HD, Fendt M, Hieke C, Heyde V, Mfune JKE, Borgemeister $C$, et al. Simultaneous circulation of two West Nile virus lineage 2 clades and Bagaza virus in the Zambezi region, Namibia. PLoS Negl Trop Dis. 2021;15:e0009311.

39. Newman CM, Cerutti F, Anderson TK, Hamer GL, Walker ED, Kitron UD, et al. Culex flavivirus and West Nile virus mosquito coinfection and positive ecological association in Chicago, United States. Vector Borne Zoonotic Dis. 2011;11:1099.

40. Kim DY, Guzman H, Bueno R, Dennett JA, Auguste AJ, Carrington CV, et al. Characterization of Culex Flavivirus (Flaviviridae) strains isolated from mosquitoes in the United States and Trinidad. Virology. 2009;386:154-159. 